\title{
Sprouty 2 protein is downregulated in human squamous cell carcinoma of the head and neck and suppresses cell proliferation in vitro
}

\author{
CHIANG-LIANG LIN ${ }^{1 *}$, WEI-FAN CHIANG ${ }^{2,3^{*}}$, CHAO-LING TUNG $^{4}$, JENG-LONG HSIEH $^{5}$, \\ JENN-REN HSIAO $^{6}$, WEN-TSUNG HUANG ${ }^{1}$, LI-YIA FENG ${ }^{7}$, CHI-HUA CHANG $^{2}$, \\ SHYUN-YEU LIU ${ }^{8}$, CHAO-JUNG TSAO ${ }^{1}$ and YIN-HSUN FENG ${ }^{4,5}$
}

\begin{abstract}
Departments of ${ }^{1}$ Hematology and Oncology, and ${ }^{2}$ Dentology, Chi-Mei Medical Center, Liouying, Tainan 73657;
${ }^{3}$ School of Dentistry, National Yang-Ming University, Taipei 11221; ${ }^{4}$ Department of Hematology and Oncology, Chi-Mei Medical Center, Tainan 71004; ${ }^{5}$ Department of Nursing, Chung Hwa University of Medical Technology, Tainan 71703; ${ }^{6}$ Department of Otolaryngology, National Cheng Kung University Hospital, Tainan 70101;

${ }^{7}$ National Kaohsiung University of Hospitality and Tourism, Kaohsiung $81271 ;{ }^{8}$ Department of

Dentistry, Chi-Mei Medical Center, Tainan 71004, Taiwan, R.O.C.
\end{abstract}

Received September 22, 2013; Accepted July 21, 2014

DOI: $10.3892 / \mathrm{mmr} .2014 .2700$

\begin{abstract}
Sprouty2 is known for its tumor-suppressing effect in various human malignant diseases. In head and neck squamous cell carcinoma (HNSCC), the role of sprouty 2 in tumorigenesis and clinical implication remains elusive. The aim of the present study was to investigate the expression of sprouty2 in patients with HNSCC and its function in vitro. Quantitative analysis of mRNA expression of sprouty2 was performed on frozen tumor samples from 42 patients with HNSCC and 19 with oral verrucous hyperplasia $(\mathrm{OVH})$ with paired counterparts of normal mucosa. Downregulation of sprouty 2 expression was demonstrated in $79 \%$ of HNSCC samples and in $58 \%$ of OVH samples compared with paired samples of normal mucosa. Enhanced expression of sprouty 2 protein suppressed the growth of HNSCC cells and signaling of the phosphorylated AKT pathway. Following transfection of the sprouty2 plasmid, HNSCC cells were more sensitive to sorafenib, a tyrosine kinase inhibitor of Raf and vascular endothelial growth factor receptor. The present study suggested that sprouty 2 expression was downregulated and behaved as a tumor suppressor in HNSCC. Sprouty2 expression in tumor cells enhanced sensitivity to sorafenib. Further studies are
\end{abstract}

Correspondence to: Dr Yin-Hsun Feng, Department of Hematology and Oncology, Chi-Mei Medical Center, 901 Chung-Hwa Road, Yong Kang District, Tainan 71004, Taiwan, R.O.C.

E-mail: yinhsun.feng@gmail.com

*Contributed equally

Key words: sprouty2, head and neck squamous cell carcinoma, sorafenib required to define the clinical impact of sprouty2 in patients with HNSCC.

\section{Introduction}

Head and neck squamous cell carcinoma (HNSCC) represents one of the six most common types of cancer in the world, with up to 500,000 novel cases annually; this includes malignancies arising in the oral cavity, larynx and pharynx. The highest rates of HNSCC are found in South-Central Asia, Central and Eastern Europe; rates are lowest in Africa and Central America, with no variation in prevalence between genders $(1,2)$. Significant risk factors for HNSCC carcinogenesis include tobacco use, alcohol consumption and infection with human papilloma virus. In Taiwan, a positive correlation has been reported between the consumption of betel nuts and incidence of HNSCC (3). Furthermore, $\sim 30 \%$ of HNSCC revealed mutations in genes that regulate squamous differentiation, including Notch1, interferon regulatory factor 6 and p63, therefore implicating their dysregulation as a major driver of HNSCC carcinogenesis (4). However, the mechanisms underlying the malignant progression of HNSCC remains to be fully elucidated.

Epidermal growth factor (EGF) receptor (EGFR) is overexpressed in $40-90 \%$ of HNSCC and is associated with a poorer outcome for HNSCC patients $(5,6)$. Ligand binding triggers the EGFR receptor to either homodimerize or heretrodimerize with other members of the Erb family, both leading to EGFR autophosphorylation. The activated receptor recruits signaling complexes and activates Ras-mitogen-activated protein kinase (MAPK), extracellular signal-regulated kinase (ERK), phosphatidynositol-3-kinase (PI3K)-Akt, signal transducers and activators of transcription and the phospholipase $\mathrm{C}$ gamma pathway (7). These unique cascades converge with the upregulation of cyclin D1, a key mediator of mitosis and G1 to S-phase progression (8). Angiogenesis has recently been recognized 
as a novel therapeutic strategy due to its important role in the pathogenesis of HNSCC. Vascular endothelial growth factors (VEGFs) and their receptors are expressed in the majority of HNSCC tumors. Several preclinical studies indicated that these markers are associated with tumor progression, changes in microvessel density and the development of lymph node metastasis $(9,10)$.

Sprouty was first reported as an antagonistic regulator of fibroblast growth factor (FGF) signaling during tracheal branching in Drosophila (11). Four mammalian sprouty genes have been defined, based on their sequence similarity with Drosophila sprouty (12). In analogy with Drosophila sprouty, mammalian sprouty proteins were proven to antagonize FGF-stimulated organogenesis (13). It was reported that the overexpression of mammalian sproutyl and sprouty 2 inhibited FGF-, VEGF- and EGF-induced proliferation and migration in human umbilical vein endothelial cells and a mouse embryonic fibroblast cell line, NIH3T3 $(14,15)$. It has become evident that expression of sprouty protein is dysregulated in numerous types of human cancer. A decrease in Sprouty2 expression has been demonstrated in hepatocellular carcinoma, lung cancer and other cancer types, this is consistent with its inhibitory on ERK1/2 signaling and proliferation $(16,17)$. A previous study by our group, identified that sprouty 2 was downregulated in colon cancer and had a tumor suppresive role (18). However, the tumor-suppressing effect of sprouty 2 in HNSCC has not yet been investigated.

Cetuximab is a well-known targeted therapy in HNSCC, which has FDA approval. In addition to EGFR targeted therapy, angiogenesis-based cancer treatment is under extensive investigation. Therapeutic strategies that have been developed in treating cancer patients include monoclonal antibodies and kinase inhibitors. VEGF activation has been demonstrated in HNSCC (19), and high VEGF expression in HNSCC tumor samples has been associated with progression of lymph node spread and poor prognosis (20). Sorafenib, also known as NSC 724772 , BAY 43-9006 or Nexavar, is a multi-targeted agent that inhibits VEGF receptor (VEGFR), platelet-derived growth factor receptor (PDGFR), KIT, fetal liver tyrosine kinase 3 (FLT-3), and the serine-threonine kinase Raf. Williamson et al (21) conducted a phase II trial of sorafenib in chemotherapy-naïve patients with recurrent or metastatic HNSCC. The median times to progression and overall survival were four and nine months, respectively. The majority of participants tolerated the sorafenib treatment well. The present study aimed to explore potential factors that may influence the response of HNSCC patients to sorafenib-based targeted therapies.

\section{Materials and methods}

Clinical specimens and cell lines. Primary HNSCC tissues and normal-appearing tissue from the head and neck, adjacent to the carcinomas, were resected from 42 patients who underwent surgery for malignant tumors. These samples were obtained from Biobank (Chi-Mei Hospital, Liouying, China) without identification of patients, following the approval of the Institutional Review Board of the Chi-Mei Hospital (Liouying, China; IRB No. CLH-0120). Written informed consent was obtained from all patients. The samples included 3 stage I, 11 stage II, 2 stage III and 26 stage IV carcinomas. Verrucous carcinoma was not included. Paired samples of verrucous hyperplasia and normal skin tissues were acquired from 19 patients who received wide excision of skin lesions from the head and neck region. Human cell lines of HNSCC were obtained from the Food Industry Research and Development Institute (Hsinchu, Taiwan) and included SCC-4 and SCC-25. Fadu cells were provided by the American Type Culture Collection (ATCC; Rockville, MD, USA). HONE-1 and HSC-3 were from Dr. Jenn-Ren Hsiao (Cheng Kung University, Tainan, Taiwan). Of these, HONE-1 was originally obtained from the ATCC and HSC-3 was obtained from the Food Industry Research and Development Institute. These cell lines were cultured in complete medium containing Dulbecco's modified Eagle's medium (DMEM) supplemented with $10 \%$ fetal bovine serum (FBS; both from Gibco Life Technologies, Darmstadt, Germany).

RNA extraction and reverse transcription. Total RNA was extracted from primary tumor tissue and normal mucosa of patients using RNA extraction kits (RNeasy Midi kit; Qiagen, Hilden, Germany). RNA was treated according to the manufacturer's instructions of the SuperScript First-Strand Synthesis System (Invitrogen Life Technologies, Carlsbad, CA, USA).

Quantitative real-time polymerase chain reaction (PCR). For each PCR reaction, $2 \mu \mathrm{g}$ of cDNA was used for PCR amplification on each sample with $12.5 \mu \mathrm{l}$ of TagMan PCR Master Mix (Applied Biosystems, Foster City, CA, USA) and $1.25 \mu \mathrm{l}$ of TagMan Assay-on-Demand kit (Applied Biosystems) that included forward primer, reverse primer and probe. Samples were run on an ABI HT7900 Sequence Detector (Applied Biosystems) according to the manufacturer's instructions. The following primers were used: human Sprouty2, 5'-GCGATCACGGAGTTCAG-3' (forward) and 5'-GTGGAGTCTCTCGTGT-3' (reverse); and human GAPDH, 5'-GGTGTGAACCATGAGAAG-3' (forward) and 5'-CCACAGTTTCCCGGAG-3' (reverse). The crossing points that were calculated with SDS software version 2.2 (Applied Biosystems) took into account the difference in amplification efficiency of the target (Sprouty2) and the reference genes. The delta-delta $\mathrm{Ct}$ assay was used for the calculation of quantitative gene expression. Fold changes in gene expression of sprouty 2 between tumor and normal tissue were calculated.

Immunoblot analysis. Cells were washed twice with phosphate-buffered saline (PBS) and harvested for immunoblot analysis. Cell lysates were prepared in the SDS sample buffer and proteins were separated by SDS-PAGE. Proteins were transferred to a nitrocellulose membrane and then blocked with $5 \%$ BSA in PBS-T $(0.1 \%$ Tween-20 in PBS). The primary antibodies were diluted according to the manufacturer's recommendation in 5\% BSA in PBS-T and incubated with the membrane at $4^{\circ} \mathrm{C}$ overnight. The antibodies used were: Rabbit anti-human polyclonal antibodies to Sprouty2 (1:2,000; Upstate Biotechnology, Temecula, CA, USA), rabbit polyclonal anti-human-actin (1:3,000; A 2668; Sigma Aldrich, St. Louis, MO, USA), rabbit anti-human monoclonal antibodies to phosphatase and tensin homolog (PTEN, 1:1,000; Epitomics, Burlingame, CA, USA), mouse anti-human monoclonal antibodies to phosphorylated Akt 

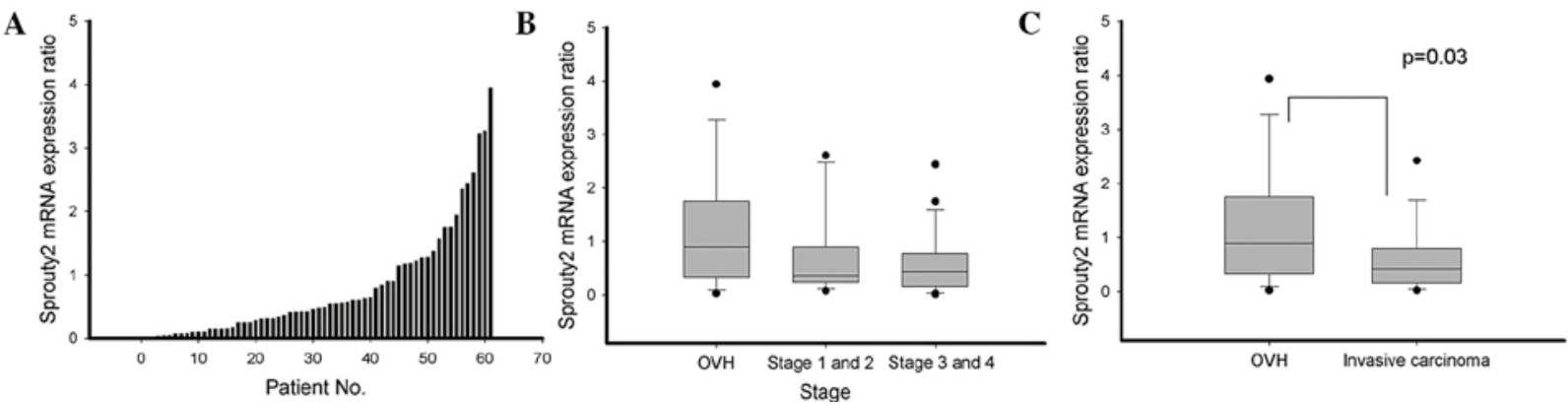

Figure 1. Sprouty2 expression is downregulated in HNSCC and OVH. The downregulation is more significant in HNSCC compared with OVH. (A) Detection of sprouty 2 gene expression in 61 human HNSCC and OVH samples with adjacent non-tumor mucosa samples by real-time quantitative polymerase chain reaction analysis. Among these patients, 19 had OVH, 14 had stage I or II HNSCC and 28 had stage III or IV HNSCC. Sprouty 2 mRNA expression in OVH or HNSCC samples relative to expression in normal tissue samples from the same patient was determined. Each sample was analyzed three times and a mean value was considered. (B) Comparison of sprouty 2 expression among patients with OVH or HNSCC with varying disease severity. P $<0.05$ between all groups. (C) The expression of sprouty2 in HNSCC was significantly lower compared with OVH. Dot, 5-95th percentile; Error bar, 10-90th percentile; Vertical box, 25-75th percentile; Horizontal bar, median. HNSCC, human head and neck squamous cell carcinoma; OVH, oral verrucous hyperplasia.

(1:1,000; Cell Signaling Technology, Inc., Beverly, MA, USA) and rabbit anti-human monoclonal antibodies to Akt (1:1,000; Cell Signaling Technology, Inc.) as primary antibodies. Horseradish peroxidase-conjugated anti-rabbit immunoglogulin G (IgG) (Santa Cruz Biotechnology, Santa Cruz, CA, USA) and anti-mouse IgG (Santa Cruz Biotechnology) were used as secondary antibodies where appropriate and protein-antibody complexes were visualized using the enhanced chemoluminescence (ECL) system (Amersham Biosciences, Piscataway, NJ, USA).

Plasmids and transfection. The pSprouty2-EGFP-N3 plasmid encoding Sprouty2-EGFP fusion protein was derived from pEGFP-N3 (Clontech, Mountain View, CA, USA). SCC-4 and HONE-1 cells were transiently transfected with pSprouty2-EGFP-N3 with Lipofectamine 2000 (Invitrogen Life Technologies). The control cells were transfected with empty vector.

In vitro proliferation assay. Cell proliferation was measured by MTT assay to assess the effect of sprouty 2 and sorafenib on the growth of human HNSCC cell lines. Cells transfected with pSprouty2-EGFP-N3 or control cells were cultured in 96-well plates (Corning Costar, Corning, NY, USA) at a density of 4,000 cells per well. Cell proliferation was determined with the MTT assay 72 hours after cell plating. The absorbance was measured at $570 \mathrm{~nm}$ using a microtiter plate reader (MRX Revelation, DYNEX, Chantilly, VA, USA). To evaluate the cytotoxic effect of sorafenib (Bayer HealthCare, Whippany, NJ, USA) influenced by sprouty 2 regulation, SCC-4 or HONE-1 cells were plated at a density of 4,000 cells per well in DMEM/F12 (Gibco Life Technologies) with $10 \%$ FBS. After 24 hours of incubation, increasing doses of sorafenib $(0,0.125,0.25,0.5,1,2,4,8$, 16 and $32 \mu \mathrm{M})$ were added to the culture medium. The MTT assay was processed as mentioned above following incubation with sorafenib for three days. The $\mathrm{IC}_{50}$ was calculated by SigmaPlot 9.0 statistical software (Systat Software, Inc., San Jose, CA, USA). Values are expressed as the ratio of cell numbers relative to the controls. Each value represents the mean \pm standard deviation (SD) of six determinations.
Statistical analysis. The Kaplan-Meier method was used to analyze time-to-event variables, and the $95 \%$ confidence interval (CI) for the median time-to-event was computed. The Student's $t$ test and paired t-test were used for the difference between groups. Any P-value of $<0.05$ was considered to indicate statistical significance. All statistical analyses were performed using SigmaPlot 9.0 statistical software (Jandel Scientific).

\section{Results}

Sprouty2 expression is downregulated in human HNSCC and oral verrucous hyperplasia $(\mathrm{OVH})$ - the downregulation was correlated with the severity of the disease. mRNA expression of sprouty 2 was determined in tumor tissues at varying disease stages and tissues from adjacent normal mucosa from 19 OVH patients and $42 \mathrm{HNSCC}$ patients by real-time quantitative PCR. Expression of sprouty2 mRNA in human tumors relative to that in their normal tissue counterparts from the same patients was compared. Overall, the mRNA expression of sprouty 2 was decreased in 44 of $61(72 \%)$ paired tumor samples compared with normal mucosal tissues. Specifically, a decrease in sprouty 2 mRNA expression was found in $58 \%$ (11/19) of OVH samples, $79 \%(11 / 14)$ of stage I-II HNSCC samples and $79 \%(22 / 28)$ of stage III-IV HNSCC samples (Fig. 1A and B). The mean sprouty 2 mRNA expression levels relative to their adjacent normal mucosa from three determinations were significantly lower in HNSCC (mean=0.634; 95\% CI=0.425-0.844) than in OVH (mean=1.164; CI=0.599-1.730; $\mathrm{P}=0.029$ ) (Fig. 1C). These findings showed that sprouty 2 was downregulated in OVH and HNSCC.

Sprouty2 expression is not associated with features of HNSCC, including tumor size, differentiation grading, nodal metastasis and recurrence - downregulation of sprouty2 has no role in the recurrence-free survival of HNSCC. The downregulation of sprouty 2 was correlated with invasiveness of HNSCC. In prostate cancer, the mean expression of sprouty2 was significantly decreased in patients experiencing recurrences during the follow-up period. The expression of sprouty2 

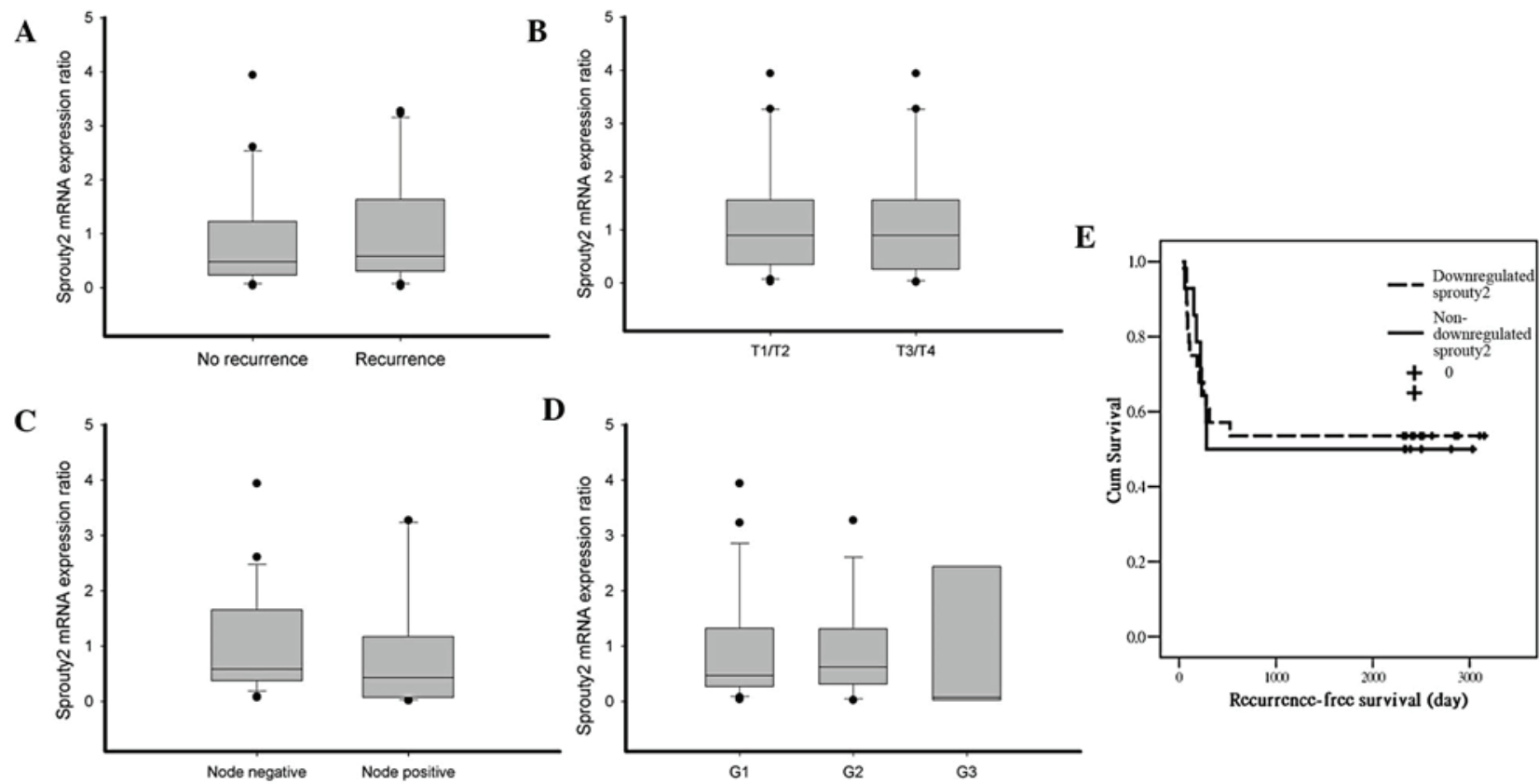

Figure 2. Sprouty2 expression is not associated with clinical features of HNSCC or survival outcomes in invasive HNSCC. Detection of sprouty2 gene expression in 42 human HNSCC samples was performed by real-time quantitative polymerase chain reaction analysis. The expression of sprouty2 gene was not statistically different by (A) recurrence status, (B) tumor size, (C) lymph node involvement or (D) histological grading. Dot, 5-95th percentile; Error bar, 10-90th percentile; Vertical box, 25-75th percentile; Horizontal bar, median. (E) Recurrence-free survival was not significantly different in HNSCC with or without downregulated sprouty2. HNSCC, head and neck squamous cell carcinoma; T, tumor size grade; G, histological grade.

tended to be decreased in advanced-stage cancers and in cases with lymph node metastasis in comparison with early stage cancer (T2 vs $>$ T2) (22). Among 42 cases of invasive HNSCC samples in the present study, the expression of sprouty $2 \mathrm{did}$ not correlate with recurrence, nodal metastasis, tumor size or histological grading (Fig. 2A-D). Song et al (23) showed that hepatoma patients negative for sprouty 2 had poorer survival. In the present study, the correlation of sprouty2 expression with recurrence-free survival was estimated by the Kaplan-Meier method in HNSCC tumors. The expression of sprouty2 was not associated with any significant changes in recurrence-free survival (Fig. 2E).

Sprouty2 enhances the expression of PTEN and unexpectedly suppresses Akt phosphorylation, which indicates the tumor suppressive role of sprouty 2 in HNSCC. Western blot analysis showed that HSC-3, HONE-1, and SCC-4, cells expressed lower levels of sprouty2 among the five HNSCC cell lines examined (Fig. 3). To verify the effect of sprouty2 in signaling pathways in HNSCC, HONE-1 and SCC-4, cells were transfected with pSprouty2-EGFP-N3 plasmid to enhance the expression of sprouty2. HSC-3 cells did not demonstrate ectopic expression of sprouty 2 due to inadequate transfection efficacy by lipofectamine. As shown in Fig. 3, the overexpression of sprouty2 reduced the phosphorylation of Akt and increased the expression PTEN. Of note, PTEN is known to be a tumor suppressor gene, implicating that sprouty2 may synergize with PTEN to suppress HNSCC cell growth $(18,24)$. Furthermore, the effect of sprouty 2 on the proliferation of HONE-1 and SCC-4 cells was investigated. Fig. 4A shows that cell proliferation, examined by the MTT assay, was decreased in both HONE-1 and SCC-4 cells overexpressing sprouty 2 compared with their
A

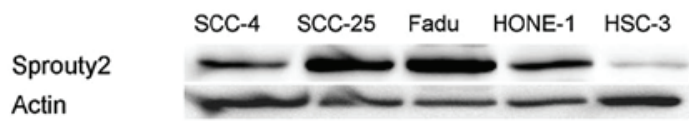

B

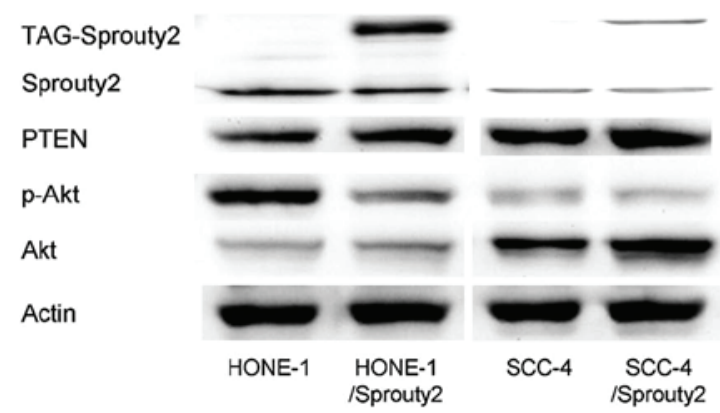

Figure 3. Sprouty2 protein expression in human HNSCC cell lines. (A) SCC-4, HONE-1 and HSC-3 had relatively low expression of sprouty2 protein compared with SCC-25 and Fadu. (B) Enhanced sprouty2 protein expression was associated with increased PTEN expression and decreased phosphorylation of Akt. The percentage of the change of target protein expression was calculated by densitometric analysis normalized to control cells. HNSCC, head and neck squamous cell carcinoma; SCC, squamous cell carcinoma; HSC, hepatic stellate cells; PTEN, phosphotase and tensin homologue gene; p-AKT, phosphorylated AKT.

control counterparts, which were transfected with empty vector $(\mathrm{P}<0.01)$.

Sprouty2 enhances the sensitivity of sorafenib in HNSCC cells, SCC-4 and HONE-1. The influence of sprouty 2 expression on the effect of sorafenib was determined. SCC-4 and HONE-1 cells were transfected with pSprouty2-EGFP-N3 

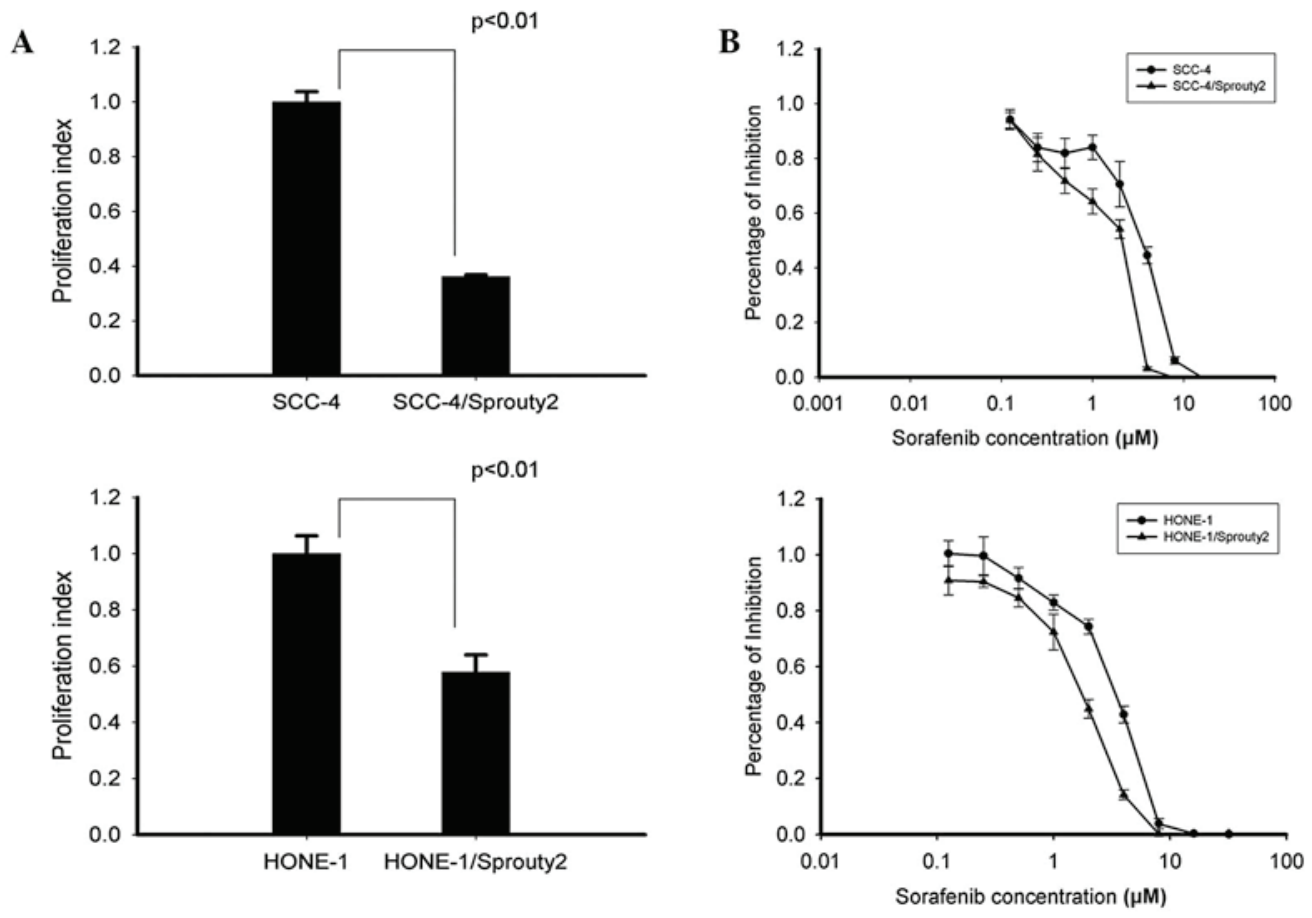

Figure 4. Sprouty2 expression inhibits the cellular proliferation in human HNSCC cells and enhances sensitivity to sorafenib. (A) Cell proliferation was measured by the MTT assay. Each point represents the mean of the determinations and error bars represent standard deviation. (n=6). SCC-4 and HONE-1 cells with enhanced expression of sprouty2 grew significantly slower compared with control cells $(\mathrm{P}<0.01)$. (B) Cytotoxic effect of sorafenib in HNSCC cells with or without sprouty2 expression. The $\mathrm{IC}_{50}$ values of sorafenib were $1.78 \pm 0.18 \mu \mathrm{M}$ in Sprouty2-SCC-4 cells and 3.54 $\pm 0.24 \mu \mathrm{M}$ in SCC-4 control cells. In Sprouty2-HONE-1 cells and HONE-1 control cells, the $\mathrm{IC}_{50}$ values of sorafenib were $1.86 \pm 0.08 \mu \mathrm{M}$ and $3.30 \pm 0.15 \mu \mathrm{M}$, respectively. Enhanced sprouty2 expression sensitized SCC-4 and HONE-1 cells to sorafenib. HNSCC, head and neck squamous cell carcinoma; SCC, squamous cell carcinoma; $\mathrm{IC}_{50}$, half maximum inhibitory concentration.

plasmid to express sprouty 2 protein. Overexpression of sprouty 2 enhanced the sensitivity of both SCC- 4 and HONE-1 cells to sorafenib compared with control cells. The half maximal inhibitory concentration $\left(\mathrm{IC}_{50}\right)$ values of sorafenib were in $3.54 \pm 0.24 \mu \mathrm{M}$ in SCC- 4 control cells and $1.78 \pm 0.18 \mu \mathrm{M}$ in SCC-4/sprouty2 cells. In HONE-1 cells, the $\mathrm{IC}_{50}$ values of sorafenib were $3.30 \pm 0.15 \mu \mathrm{M}$ in control cells and $1.86 \pm 0.08 \mu \mathrm{M}$ in sprouty2-transfected cells.

\section{Discussion}

Sprouty proteins have been identified as modulators of receptor tyrosine kinase signaling in normal development and disease states (25). Evidence suggested that the expression of sprouty proteins is dysregulated in a number of cancers; in most types of cancer it is downregulated, with the exception of colon cancer and melanoma, where it is upregulated (Table I) $(16-18,22,23,26-37)$. In prostate cancer, downregulated sprouty 2 expression in invasive prostate cancer cell lines and high-grade clinical prostate cancer is similar to that of benign prostatic hyperplasia and well-differentiated tumors (32). A previous study by our group disclosed that sprouty 2 was deregulated in human colon cancer and associated with inverse regulation by microRNA 21 (18). It implied that deregulation of sprouty 2 expression may promote tumorigenesis. To date, it has remained elusive whether sprouty 2 acts as a negative inhibitor of growth factors in HNSCC. The present study confirmed that sprouty 2 was downregulated in preneoplasia and HNSCC. Importantly, it was identified that the decrease in sprouty 2 expression in HNSCC was significant compared with $\mathrm{OVH}$, a precursor of verrucous carcinoma. It is noteworthy that sprouty 2 expression decreased in $58 \%$ of OVH samples, but was significantly decreased in invasive carcinoma. This result suggested that the decrease of sprouty 2 expression may be an early event in the carcinogenesis of HNSCC.

Previous studies on human cancers suggested the prognostic impact of sprouty2. In a hepatoma study, Lee et al (30) found that significant downregulation of sprouty 2 protein and sprouty 2 mRNA expression characterized most hepatomas with poorer outcomes. Differentiation grading is a strong predictor for prognosis in most types of cancers. Sprouty 2 protein has been found to be associated with high-grade cancers. In human glioma, sprouty 2 protein levels were significantly decreased in $79 \%$ of invasive World Health Organization (WHO) grade II-IV tumors, but not in non-invasive WHO grade I tumors and normal tissues (28). Furthermore, by assessing the expression of sprouty 2 mRNA in resected prostate cancer, McKie et al (34) reported a trend between downregulated sprouty 2 mRNA expression and increasing Gleason score (less differentiated) tumors. The present study focused on invasive HNSCC and did not suggest sprouty 2 was a prognostic predictor, in part due to the limited number of stage I and stage II samples. Only fourteen tumor samples of stage I and stage II disease were analyzed in the present study. To verify the conclusion, the inclusion of a larger number of stage I and stage II disease samples would be required. 
Table I. Sprouty2 expression in human cancer disease.

\begin{tabular}{|c|c|c|}
\hline Cancer type & Sample origin & First author, year (reference) \\
\hline \multicolumn{3}{|l|}{ Sprouty2 downregulated cancer } \\
\hline Breast cancer & Clinical human tissue & Lo et al, 2004 (24) \\
\hline Colon cancer & Clinical human tissue & Feng et al, 2011 (18) \\
\hline Endometrial cancer & Clinical human tissue & Velasco et al, 2011 (25) \\
\hline Glioma & Clinical human tissue and cell lines & Kwak et al, 2011 (26) \\
\hline $\begin{array}{l}\text { Squamous cell carcinoma of the } \\
\text { head and neck }\end{array}$ & Clinical human tissue & Present study \\
\hline Hepatocellular carcinoma & Clinical human tissue and cell lines & $\begin{array}{l}\text { Fong et al, } 2006(17) \\
\text { Lee } \text { et al, } 2008(27) \\
\text { Lee } \text { et al, } 2010(28) \\
\text { Song } \text { et al, } 2012(22)\end{array}$ \\
\hline Lymphoma & Cell lines & Frank et al, 2009 (29) \\
\hline Non-small cell lung cancer & Clinical human tissue and cell lines & Sutterlüty et al, 2007 (16) \\
\hline Neuroblastoma & Cell lines & Ishida et al, 2007 (30) \\
\hline Pancreatic cancer & Clinical human tissue and cell lines & Ma et al, $2010(31)$ \\
\hline Prostate cancer & Clinical human tissue & $\begin{array}{l}\text { McKie et al, } 2005 \text { (32) } \\
\text { Fritzsche } \text { et al, } 2006 \text { (21) }\end{array}$ \\
\hline \multicolumn{3}{|l|}{ Sprouty2 upregulated cancer } \\
\hline Colon cancer & Clinical human tissue and cell lines & $\begin{array}{l}\text { Holgren et al, } 2010 \text { (33) } \\
\text { Barbáchano et al, } 2010 \text { (34) }\end{array}$ \\
\hline Melanoma & Cell lines & Bloethner et al, 2005 (35) \\
\hline
\end{tabular}

Sprouty proteins have been demonstrated to inhibit signaling by a diverse range of stimuli, including VEGF, PDGFR, hepatocyte growth factor, glial-derived growth factor and nerve growth factor $(38,39)$. The inhibitory function of sprouty is mainly associated with the control of the Ras/Raf/MAPK/ERK pathway at multiple levels (25). Sprouty2 interacts with different components of the EGF signaling pathway. Sprouty2 interacts with E3 ubiquitin ligase/c-Casitas B-lineage lymphoma (c-Cbl), which leads to the reduction of EGFR endocytosis and degradation (40). Thus, sprouty2 serves as a negative regulator of FGF and other growth factors but as a positive enhancer of EGF signaling. Enhanced sprouty2 protein expression has been shown to suppress Akt phosphorylation and promote PTEN expression in cancer cells $(18,24)$. In HNSCC cell lines, HONE-1 and SCC-4, ectopic expression of sprouty 2 exerted a similar regulation pattern of signaling pathways. The present study showed that the expression of sprouty 2 protein suppressed Akt phosphorylation and enhanced PTEN expression. This correlated with the tumor-suppressive effect of sprouty 2 in HNSCC.

Several mechanisms have been proposed to define the decrease of sprouty 2 in cancer cells, including the methylation of the sprouty 2 promoter, increasing degradation of sprouty2 by neural precursor cell expressed developmentally down-regulated 4 (NEDD4) and upregulation of microRNA-21. McKie et al (34) found the suppression of sprouty2 expression correlated with methylation of the $\mathrm{CpG}$ region in clinical samples of prostate cancer. Sprouty2 inactivation by promoter methylation in human B-cell lymphoma was suggested by Frank et al (31). Sprouty2 can be proteolytically degraded by c-Cbl, seven in absentia homolog-2 (SIAH-2), or NEDD4 $(30,40)$. In a hepatoma study, all 13 hepatoma samples exhibiting NEDD4 upregulation displayed low sprouty2 protein expression (30). Furthermore, upregulated microRNA-21 was associated with a decrease in sprouty2 protein levels in glioma cells (28) and reduced sprouty 2 mRNA expression in colon cancer (18), implying that the deregulation of sprouty2 is triggered differently depending on cancer biology. Further studies are warranted to address the mechanisms of deregulated sprouty 2 in HNSCC.

Sorafenib is an oral inhibitor of serine/threonine kinases C-Raf and B-Raf and the receptor tyrosine kinases VEGFR-2, VEGFR-3, PDGFR, FLT-3 and KIT. It inhibits cancer cell activity via two major pathways: The EGFR/Ras/Raf/MEK/ERK signaling pathway, which is a key regulator in cancer biology, and the VEGF/VEGFR pathway, which is essential for angiogenesis $(41,42)$. Sorafenib has been applied clinically for the treatment of patients with advanced hepatocellular carcinoma. Combining antiangiogenics to overcome resistance of anticancer therapy poses a promising strategy in the future of cancer medicine. In HNSCC, antiangiogenic agents, including bevacizumab and sorafenib, have been integrated into the treatment strategies but none of these agents have reached approval in clinical practice, despite extensive clinical studies. The proposed mechanisms of sorafenib resistance include EGFR activation (43), multidrug resistance protein 2 expression (44) and the activation of the PI3K/Akt signaling pathway in hepatocellular carcinoma (45). Accurate predictors of patient response to anticancer therapy would minimize the use of ineffective and expensive treatment with possible 
toxic side effects. Previous research efforts have focused on 'forward-signaling' mechanisms of pathway activation and have implied that the loss of negative feedback control can also lead to aberrant pathway activation. PTEN, a well-known tumor suppressor gene, has been extensively studied in EGFR inhibition of colon cancer therapy as well as trastuzumab treatment in breast cancer. Sprouty 2 protein has been shown to be involved in trastuzumab resistance in breast cancer (46) and gefitinib resistance in colon cancer cells (47). The ability of sprouty 2 to suppress Akt phosphorylation and modulate EGFR activation has encouraged researchers to explore the role of sprouty 2 in altering the effectiveness of sorafenib in HNSCC. The present study indicated that the overexpression of sprouty 2 protein suppressed HNSCC proliferation and enhanced sensitivity to sorafenib. Therefore, sprouty 2 provides a potential target to increase sorafenib effectiveness in clinical practice. However, further investigations and validation through clinical trials are required prior to application of sprouty 2 as a novel treatment for cancer patients in the clinic.

In conclusion, the present study suggested that sprouty 2 was downregulated in a high proportion of OVH and HNSCC. Sprouty2 had a negative feedback role in the suppression of Akt phosphorylation and upregulation of PTEN, which had an inhibitory effect on cell proliferation. Sprouty 2 may serve as a potential target in HNSCC treatment and a predictor of response to sorafenib therapy.

\section{Acknowledgements}

This work was supported by grants from Chi-Mei Medical Center (nos. CMNCKU10004, CMFHR10006, CLFHR9905 and CLFHR0014) and Chung Hwa University of Medical Technology (no. HWAI10003001). Thanks for the samples provided by Biobank of Chi-Mei Medical Center, Liouying. The results have been reported at the annual meeting of the American Society of Oncology, 2013.

\section{References}

1. Jemal A, Siegel R, Ward E, et al: Cancer statistics, 2008. CA Cancer J Clin 58: 71-96, 2008.

2. Jemal A, Bray F, Center MM, Ferlay J, Ward E and Forman D: Global cancer statistics. CA Cancer J Clin 61: 69-90, 2011.

3. Ko YC, Huang YL, Lee CH, Chen MJ, Lin LM and Tsai CC: Betel quid chewing, cigarette smoking and alcohol consumption related to oral cancer in Taiwan. J Oral Pathol Med 24: 450-453, 1995.

4. Stransky N, Egloff AM, Tward AD, et al: The mutational landscape of head and neck squamous cell carcinoma. Science 333: 1157-1160, 2011.

5. Ang KK, Berkey BA, Tu X, et al: Impact of epidermal growth factor receptor expression on survival and pattern of relapse in patients with advanced head and neck carcinoma. Cancer Res 62: 7350-7356, 2002.

6. Rubin Grandis J, Melhem MF, Gooding WE, et al: Levels of TGF-alpha and EGFR protein in head and neck squamous cell carcinoma and patient survival. J Natl Cancer Inst 90: 824-832, 1998.

7. KalyankrishnaS andGrandis JR:Epidermalgrowthfactorreceptor biology in head and neck cancer. J Clin Oncol 24: 2666-2672, 2006.

8. Sharafinski ME, Ferris RL, Ferrone S and Grandis JR: Epidermal growth factor receptor targeted therapy of squamous cell carcinoma of the head and neck. Head Neck 32: 1412-1421, 2010.
9. Lalla RV, Boisoneau DS, Spiro JD and Kreutzer DL: Expression of vascular endothelial growth factor receptors on tumor cells in head and neck squamous cell carcinoma. Arch Otolaryngol Head Neck Surg 129: 882-888, 2003.

10. Neuchrist C, Erovic BM, Handisurya A, et al: Vascular endothelial growth factor $\mathrm{C}$ and vascular endothelial growth factor receptor 3 expression in squamous cell carcinomas of the head and neck. Head Neck 25: 464-474, 2003.

11. Hacohen N, Kramer S, Sutherland D, Hiromi Y and Krasnow MA: Sprouty encodes a novel antagonist of FGF signaling that patterns apical branching of the Drosophila airways. Cell 92: 253-263, 1998

12. Tefft JD, Lee M, Smith S, et al: Conserved function of mSpry-2, a murine homolog of Drosophila sprouty, which negatively modulates respiratory organogenesis. Curr Biol 9: 219-222, 1999.

13. Minowada G, Jarvis LA, Chi CL, et al: Vertebrate Sprouty genes are induced by FGF signaling and can cause chondrodysplasia when overexpressed. Development 126: 4465-4475, 1999.

14. Impagnatiello MA, Weitzer S, Gannon G, Compagni A, Cotten $\mathrm{M}$ and Christofori G: Mammalian sprouty-1 and -2 are membrane-anchored phosphoprotein inhibitors of growth factor signaling in endothelial cells. J Cell Biol 152: 1087-1098, 2001.

15. Gross I, Bassit B, Benezra M and Licht JD: Mammalian sprouty proteins inhibit cell growth and differentiation by preventing ras activation. J Biol Chem 276: 46460-46468, 2001.

16. Sutterlüty H, Mayer CE, Setinek U, et al: Down-regulation of Sprouty2 in non-small cell lung cancer contributes to tumor malignancy via extracellular signal-regulated kinase pathway-dependent and -independent mechanisms. Mol Cancer Res 5: 509-520, 2007.

17. Fong CW, Chua MS, McKie AB, et al: Sprouty 2, an inhibitor of mitogen-activated protein kinase signaling, is down-regulated in hepatocellular carcinoma. Cancer Res 66: 2048-2058, 2006.

18. Feng YH, Wu CL, Tsao CJ, et al: Deregulated expression of sprouty 2 and microRNA-21 in human colon cancer: Correlation with the clinical stage of the disease. Cancer Biol Ther 11: 111-121, 2011.

19. Bancroft CC, Chen Z, Dong G, et al: Coexpression of proangiogenic factors IL-8 and VEGF by human head and neck squamous cell carcinoma involves coactivation by MEK-MAPK and IKK-NF-kappaB signal pathways. Clin Cancer Res 7: 435-442, 2001.

20. Mineta H, Miura K, Ogino T, et al: Prognostic value of vascular endothelial growth factor (VEGF) in head and neck squamous cell carcinomas. Br J Cancer 83: 775-781, 2000.

21. Williamson SK, Moon J, Huang CH, Guaglianone PP, LeBlanc M, Wolf GT et al. Phase II evaluation of sorafenib in advanced and metastatic squamous cell carcinoma of the head and neck: Southwest Oncology Group Study S0420. J Clin Oncol. 2010;28(20):3330-5.

22. Fritzsche S, Kenzelmann M, Hoffmann MJ, et al: Concomitant down-regulation of SPRY1 and SPRY2 in prostate carcinoma. Endocr Relat Cancer 13: 839-849, 2006.

23. Song K, Gao Q, Zhou J, et al: Prognostic significance and clinical relevance of Sprouty 2 protein expression in human hepatocellular carcinoma. Hepatobiliary Pancreat Dis Int 11: 177-184, 2012.

24. Edwin F, Singh R, Endersby R, Baker SJ and Patel TB: The tumor suppressor PTEN is necessary for human Sprouty 2-mediated inhibition of cell proliferation. J Biol Chem 281: 4816-4822, 2006.

25. Edwin F, Anderson K, Ying C and Patel TB: Intermolecular interactions of Sprouty proteins and their implications in development and disease. Mol Pharmacol 76: 679-691 2009.

26. Lo TL, Yusoff P, Fong CW, et al: The ras/mitogen-activated protein kinase pathway inhibitor and likely tumor suppressor proteins, sprouty 1 and sprouty 2 are deregulated in breast cancer. Cancer Res 64: 6127-6136, 2004.

27. Velasco A, Pallares J, Santacana M, et al: Promoter hypermethylation and expression of sprouty 2 in endometrial carcinoma. Hum Pathol 42: 185-193, 2011.

28. Kwak HJ, Kim YJ, Chun KR, et al: Downregulation of Spry2 by miR-21 triggers malignancy in human gliomas. Oncogene 30 : 2433-2442, 2011

29. Lee SA, Ho C, Roy R, et al: Integration of genomic analysis and in vivo transfection to identify sprouty 2 as a candidate tumor suppressor in liver cancer. Hepatology 47: 1200-1210, 2008.

30. Lee SA, Ladu S, Evert M, et al: Synergistic role of Sprouty2 inactivation and c-Met up-regulation in mouse and human hepatocarcinogenesis. Hepatology 52: 506-517, 2010. 
31. Frank MJ, Dawson DW, Bensinger SJ, et al: Expression of sprouty 2 inhibits B-cell proliferation and is epigenetically silenced in mouse and human B-cell lymphomas. Blood 113: 2478-2487, 2009.

32. Ishida M, Ichihara M, Mii S, et al: Sprouty2 regulates growth and differentiation of human neuroblastoma cells through RET tyrosine kinase. Cancer Sci 98: 815-821, 2007.

33. Ma Y, Yu S, Zhao W, Lu Z and Chen J: miR-27a regulates the growth, colony formation and migration of pancreatic cancer cells by targeting Sprouty2. Cancer Lett 298: 150-158, 2010

34. McKie AB, Douglas DA, Olijslagers S, et al: Epigenetic inactivation of the human sprouty2 (hSPRY2) homologue in prostate cancer. Oncogene 24: 2166-2174, 2005.

35. Holgren C, Dougherty U, Edwin F, et al: Sprouty-2 controls c-Met expression and metastatic potential of colon cancer cells: sprouty/c-Met upregulation in human colonic adenocarcinomas. Oncogene 29: 5241-5253, 2010.

36. Barbáchano A, Ordóñez-Morán P, García JM, et al: SPROUTY-2 and E-cadherin regulate reciprocally and dictate colon cancer cell tumourigenicity. Oncogene 29: 4800-4813, 2010.

37. Bloethner S, Chen B, Hemminki K, et al: Effect of common B-RAF and N-RAS mutations on global gene expression in melanoma cell lines. Carcinogenesis 26: 1224-1232, 2005

38. Sasaki A, Taketomi T, Kato R, et al: Mammalian Sprouty4 suppresses Ras-independent ERK activation by binding to Raf1. Nat Cell Biol 5: 427-432, 2003.

39. Mason JM, Morrison DJ, Basson MA and Licht JD: Sprouty proteins: multifaceted negative-feedback regulators of receptor tyrosine kinase signaling. Trends Cell Biol 16: 45-54, 2006.
40. Wong ES, Fong CW, Lim J, et al: Sprouty2 attenuates epidermal grow th factor receptor ubiquitylation and endocytosis, and consequently enhances Ras/ERK signalling. EMBO J 21: 4796-4808, 2002.

41. Wilhelm SM, Carter C, Tang L, et al: BAY 43-9006 exhibits broad spectrum oral antitumor activity and targets the $\mathrm{RAF} / \mathrm{MEK} / \mathrm{ERK}$ pathway and receptor tyrosine kinases involved in tumor progression and angiogenesis. Cancer Res 64: 70997109, 2004.

42. Chang YS, Adnane J, Trail PA, et al: Sorafenib (BAY 43-9006) inhibits tumor growth and vascularization and induces tumor apoptosis and hypoxia in RCC xenograft models. Cancer Chemother Pharmacol 59: 561-574, 2007.

43. Ezzoukhry Z, Louandre C, Trécherel E, et al: EGFR activation is a potential determinant of primary resistance of hepatocellular carcinoma cells to sorafenib. Int J Cancer 131: 2961-2969, 2012.

44. Shibayama Y, Nakano K, Maeda H, et al: Multidrug resistance protein 2 implicates anticancer drug-resistance to sorafenib. Biol Pharm Bull 34: 433-435, 2011.

45. Chen KF, Chen HL, Tai WT, et al: Activation of phosphatidylinositol 3-kinase/Akt signaling pathway mediates acquired resistance to sorafenib in hepatocellular carcinoma cells. J Pharmacol Exp Ther 337: 155-161, 2011.

46. Faratian D, Sims AH, Mullen P, et al: Sprouty 2 is an independent prognostic factor in breast cancer and may be useful in stratifying patients for trastuzumab therapy. PLoS One 6: e23772, 2011.

47. Feng YH, Tsao CJ, Wu CL, et al: Sprouty2 protein enhances the response to gefitinib through epidermal growth factor receptor in colon cancer cells. Cancer Sci 101: 2033-2038, 2010. 